\title{
VALIDATION OF THE NEW VISUAL SWIMMIMG PACE CONTROL SYSTEM IN REAL-TIME
}

\author{
Stefan Szczepan, ${ }^{A, B, C, D, E}$ Krystyna Zaton ${ }^{A, B, E}$ \\ Faculty of Physical Education, Department of Swimming, University School of Physical Education, Wroclaw, Poland \\ A Study Design; ${ }^{\mathrm{B}}$ Data Collection; ${ }^{\mathrm{C}}$ Statistical Analysis; ${ }^{\mathrm{D}}$ Manuscript Preparation; ${ }^{\mathrm{E}}$ Funds Collection \\ Address for correspondence: \\ Stefan Szczepan \\ Department of Swimming, University School of Physical Education in Wroclaw, Poland \\ I.J. Paderewskiego 35, 51-612 Wrocław, Poland \\ E-mail: stefan.szczepan@awf.wroc.pl
}

\begin{abstract}
Ahstract Controlling swimming speed, i.e. the intensity of physical activity, is an important factor in swimming training. The aim of this study was to validate the new "Swimming Pace Control System" (SPCS) for the control of swimming speed in real time using visual information. Submerged at the bottom of the pool was a system equipped with LEDs and software that informed the swimmer of the appropriate distance and swimming speed. A validation test was completed with an accuracy of $\pm 200 \mathrm{~ms}$ which compared the predetermined time for the beam of light emitted by the SPCS and the times achieved and recorded by the electronic starting system; "Colorado Time System" (Colorado Time, USA). The average time required to move the beam simulated by the SPCS at fixed distances $(150 \mathrm{~m}, 100 \mathrm{~m}, 50 \mathrm{~m})$ was within the assumed error of measurement (500 ms). SPCS was proven to be useful for control of swimming speed in real-time with the aid of visual information. The system gives an objective indication of swimming speed, thus it can be used in swimming training and during empirical research.
\end{abstract}

Key WOrlls visual information, swimming speed, speed control system, device validation

\section{Introduction}

Objective assessment of the achievements of athletes is a popular topic (Toussaint, Truijens, 2005). With scientific and technological progress there comes the growing possibility of obtaining feedback in order to assess technical capacity and endurance. This then leads to improvements in the performance of athletes across a range of different disciplines.

The need for measuring and evaluating numerous technical parameters and physiological forces is a means of creating improved diagnostic methods and measuring devices. The focal parameters where improvements are highly sought-after are diagnostic evaluation, technique, and stamina. A multitude of methods for recording and quantifying technical data and movement provides trainers and researchers with many possibilities, which in turn results in improved athletic performance. 
The origins of evaluation techniques were primarily based on the method of photographic film, which allowed for the opportunity to register the displacement of a point positioned on the body of a subject in time and space (Lees, 2002). Nowadays, contemporary methods allow for film recordings of movements with high frequency footage (up to 1000 frames/s). In the last two decades the most prevalent method for assessing the displacement of the body in space and time has proven to be measurements based on computer programs (Tanabe, Ito, 2007). Typical examples include traffic analysis software, such as Dartfish (Dartfish HQ, CH), or SIMI Motion2D (Reality Motion Systems 2D GmbH, DE). We may also observe the use of more advanced means, such as telemetry data acquisition systems like Qualisys (Qualisys $A B, S E$ ). Such methods allow for the precise acquisition and further analysis of the data of an executed movement.

In the assessment of physiological parameters such as $\mathrm{Hr}$, La, and $\mathrm{VO}_{2}$ the most commonly used devices Sport-tester (Polar Electro, FI), Lactate scout (SensLab, DE), K4b2 (Cosmed, IT) - are tools that are simple to use and widely available.

Usually, the evaluation is concerned with the structure of the movement based on several types of feedback: speed, time, acceleration, angular velocity, and angle of the joints. Additional analysis requires the use of video and computer equipment. However, there is a significant delay between the completed movement and the time that the data is collected and processed, e.g. when using movement analysis software (Hohmann, Fehr, Kirsten, Krueger, 2008). Hence producers, in cooperation with practitioners, have sought to develop an assessment technique that functions while a given motor skill is in operation (Eskofier, Kugler, Melzer, Kuehner, 2012).

For actions completed on land, measurement of movement and physiological parameters of the human body and its pairing with instantaneous feedback is currently available (Chow, Carlton, Ekkekakis, Hay, 2000). However, in an aquatic environment, numerous barriers interfere with communication devices, often completely preventing the use of such tools. One example is the use of infra-red analysis of the swimming movement, which is not effective due to the density of water. In the case of assessing physiological parameters, systems have been adapted for testing respiratory endurance and circulatory status, e.g. K4b2 (Fernandes et al., 2003). With this in mind, some waterproof sport-testers are currently available.

Despite the many problems associated with methods for measuring the kinematic parameters and physiological parameters of swimmers, there are many works depicting methods of assessing the parameters of the swimming cycle while in the water. Ohgi (2002) estimated fatigue among swimmers using a motion sensor positioned on the wrist. Davey, Anderson, James (2005) used a sensor on swimmers' hips to assess stroke length. Slawson et al. (2008) and Callaway, Cobb, Jones (2009) used an accelerometer to assess the four swimming techniques. Bächlin, Tröster (2012) developed a model of swimming technique and method of measuring the overall effectiveness of swimming with the use of the accelerometer.

These researchers have formed a range of devices that support swimmers in various aspects of their training. An example of this includes a device for wireless communication with the swimmer (Zatoń, Szczepan, 2014), which improves the quality and flow of verbal information between the coach and the swimmer. Also, a visual means for the transmission of information via a timer submerged at the bottom of the pool has been used (Gonzalez et al., 2002; Perez, Llana, Brizuela, Encarnación, 2009). The presented methods of communication with swimmers, when combined with movement and physiological parameters assessment, have demonstrably helped in improving athletic achievement levels. 
For swimmers there are only a few devices available commercially which inform about swim times. Devices such as Lap Track (Finis, USA) are equipped with a display that is placed on the pool wall, or a hand chronometer (SportCount Chrono, USA).

Other devices such as Lider (Kuca, PL), GBK-Pacer (GBK-Electronics, PT), Pace2Swim (FADEUP Porto, PT), and SwimLead (Synerte, PL) report the speed of swimming in real time, using a beam of light moving along the bottom of the swimming pool. These are examples of the use of visual information, which have all been found to contribute to the improved execution of physical activities (Seat, Wrisberg, 1990; Proteau, Isabelle, 2002).

This paper presents a test of the SPCS for the control of swim speed in real time with the aid of visual information. This is another device based on new technological solutions. The transmission device communicates visual information in real time to the swimmer regarding the swim speed by means of a beam of light tracking along the bottom of the pool. It can be used in swimming training and for research purposes. This is a departure from traditional methods using a chronometer, and represents a transition to the most modern forms of measuring movement.

\section{Reasons for controlling swimming speed}

The ability to control the speed of swimming, that is, the intensity of the effort, can be seen as multifaceted (Szczepan, Zatoń, Klarowicz, 2016).

The first aspect is to increase the chance of a faster adaptation to the level of physical activity during swimming training (Costill et al., 1991; Pelayo, 1995). One example is a swimming speed within pre-designated zones which determine the intensity of the training exercise. For example, movement intensity above or below the anaerobic threshold, which is used for the adaptation process of an athlete to the appropriate concentration of lactate in the blood (Costill et al., 1991; Pérez, Llana, Brizuela, Encarnación, 2009; Scruton et al., 2015). Typically, inexperienced swimmers do not have the ability to maintain adequate swimming speed. Hence, controlling the speed enables the realization of training tasks at appropriate intensities and consequently a faster adaptation to exercise. Maintaining the correct speed is also important during recreational swimming training (aerobic training zone), for health purposes such as cardio training or swimming in order to reduce body fat.

The second aspect is improved swimming efficiency. This is made possible with the help of a stabilized swimming speed, which lowers the physiological cost incurred during exercise (Barbosa et al., 2005). Controlling and maintaining the desired swimming speed helps conserve energy required in order to perform the exercise. As a result, the swimmer's body becomes able to travel longer distances (Åstrand, Rodahl, Dahl, Stromme, 2003; Wilmore, Costill, Kenney, 2008).

The third aspect is the standard mastering of swimming techniques at various exercise intensities. This is primarily important at higher intensities, when a reduction in the precision level of the movement often occurs as it is more challenging under these circumstances to maintain exemplary technique.

The fourth aspect is an increase in the economization of swimming (expressed as speed) by optimizing stroke length and stroke rate. The stroke length and frequency of swimming movements in relation to swimming speed are objective means for the assessment of swimming techniques. Optimal relationships between stroke length and movement frequency at a given speed increases the effectiveness of swimming (expressed in speed), as well as economizing swimming techniques (expressed at the expense of physiological exertion) (Hay, 2002; Zamparo, Pendergast, Mollendorf, Termin, Minetti, 2005). 
These arguments constitute hard evidence for the importance of controlling swimming speed. Hence the need for creating a device which may be used for this purpose.

The aim of this study was to validate the new "Swimming Pace Control System" (SPCS) for the control of swimming speed in real time using visual information. It was hypothesized that the SPCS objectively indicates the speed of swimming and is available for use in training and during empirical research. The Ethics Committee of the University approved the conduct of the study.

\section{Methods}

The new SPCS was subjected to validation testing. Immersed at the bottom of the swimming pool was a system equipped with LEDs and also controlling software that indicated to the swimmer over a designated distance the appropriate swimming speed. Figures 1 and 2 show a visualization and operational overview of the system. Below is a detailed description of the device.

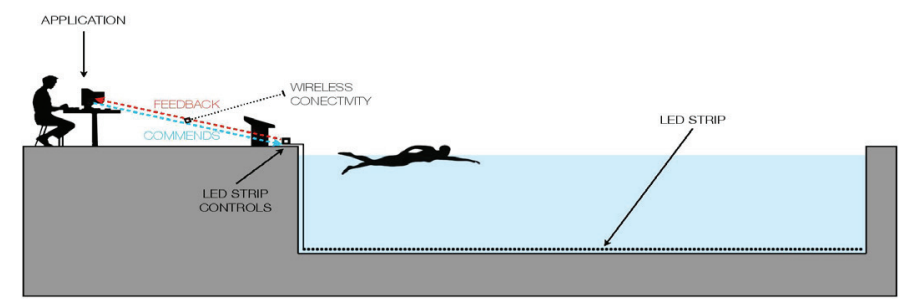

Figure 1. Visualization of the system

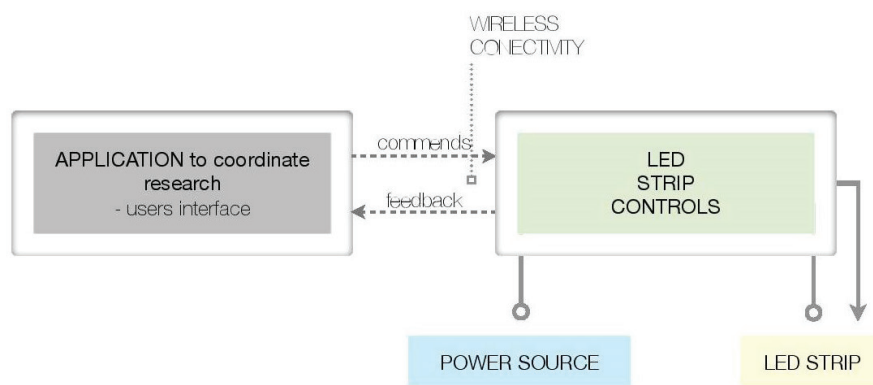

Figure 2. Schematic overview of the system

\section{Construction of the System}

SPCS consists of the following elements:

1. Waterproof LED tape:

- 750 LED RGB with 250 sliding registers WS2811 (1 register for 3 LED RGB), 
- transparent PVC pipe with a diameter of $19 \mathrm{~mm}$,

- specialized waterproof heat shrink tube with glue,

- waterproof connectors for PVC pipe,

- mains power supply,

- M12 connectors to the controller,

- weights with special handles,

- reel for winding and moving the LED tape (Figure 3).

2. The Light Signal Controller:

- the microcontroller ATmega328, is a timed system with a signal frequency of $16 \mathrm{MHz}$, which has $32 \mathrm{kB}$ of program memory Flash and $2 \mathrm{kB}$ of SRAM memory,

- bluetooth SPP visible to the system as a virtual COM port with auto speed detection, serial transmission and a control DTR/RTS/DSR. This creates the possibility of remotely controlling the system from anywhere within a $10 \mathrm{~m}$ radius,

- software microcontroller for the light signals and remote communication.

3. Device with software for controlling the tests:

- device for the operating software and data storage,

- software for the Windows 10 platform, written in the standard Universal Windows Platform; as a result the application code is able to function on a broad range of devices,

- software for the Android platform,

- software for the iOS platform (Figure 4).

4. Additional elements:

- chest,

- battery,

- cables and Connectors.

\section{Functionality}

The prototype constructed has the following functions:

1. Defining the training:

- defining the distance to complete. The distance is given as a multiple of $25 \mathrm{~m}$,

- defining the time to cover a given distance. The minimum time was set at 10 seconds for a distance of $25 \mathrm{~m}$. Time can be set with an accuracy to the nearest full second,

- define the number of series,

- defining the rest time between sets.

2. Control of light signal:

- start light signal,

- stop light signal,

- resume light signal,

- cancel light signal.

3. Presentation of visual information from the LED tape:

- the pre-start pulsing of the LED, 
- fixed rate and distance,

- the number of completed lengths of the pool,

- information on the status of the swimmer (ready to start, swimming, resting, resumed, terminated training),

- the possibility of acceleration and deceleration of the LEDs is recurring.

The system supports up to five swimmers at a time. Each swimmer is assigned a unique color so it is possible to easily keep track of the light signal under the water.

The system has two modes of operation:

- fixed intensity (primary) - set distance and time to overcome the distance (e.g. $200 \mathrm{~m} / 2$ : 30"),

- constant intensity (advanced) - measured distances and the number of repetitions, along with the time to complete them and the length of the pause between segments (e.g. $10 \times 100 \mathrm{~m} / 1: 30$ "/rest 30") - the ability to set a break for 0 sec, allowing a start, for example, at $1: 30$ ".

\section{Non-functional requirements}

Waterproof

One of the most important non-functional requirements is the waterproofing of the LED tape. This requirement is met by the use of specialized PVC pipes, connectors and waterproof heat-shrink tubing for industrial applications. Test sessions revealed that the design solution and the construction itself are fully waterproof.

Accuracy of time over a given distance

In the analysis phase of the project an error accuracy range was set for the time to complete a given distance of $\pm 200 \mathrm{~ms}$. Test sessions have shown that this requirement is met.

Figure 3 shows the LED tape wound on a reel before installation at the bottom of the swimming pool.

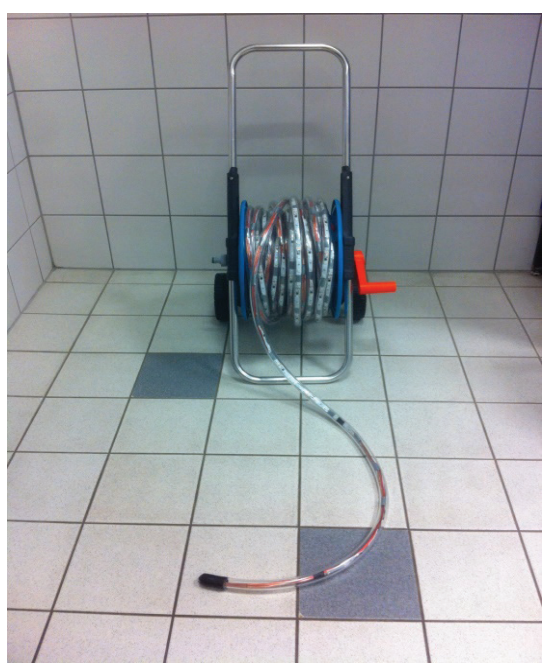

Figure 3. LED tape before installation at the bottom of the swimming pool 
Figure 4 shows an overview of software for the control system.

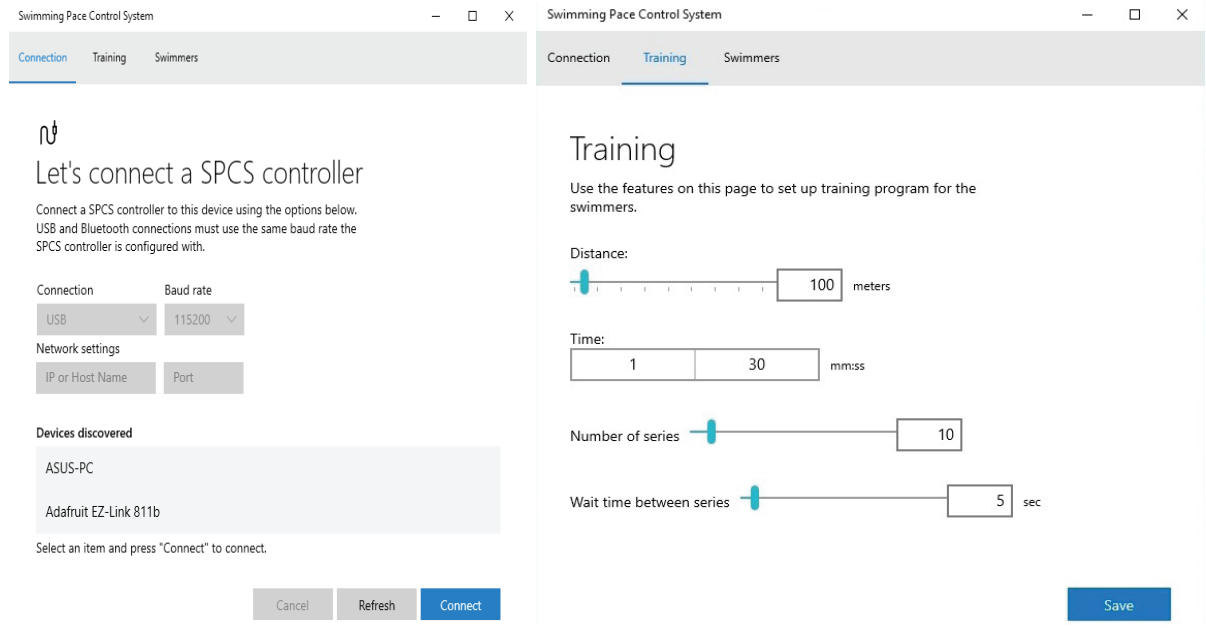

Figule 4. The application control system

\section{Algorithms}

A key part of the software is an algorithm for calculating the cycle time for the refresh position of the LED light on the tape. Cycle value provides an accurate reflection of the preset time to complete the distance.

The basic formula for calculating the cycle is as follows:

$$
\text { Cycle time }=\frac{\frac{\text { fixed time }}{\text { total lenghts }}}{\text { number of LED }} \times 1000-\text { LED chargetime. }
$$

Fixed time - is the time to complete the distance as specified by the user at the moment of defining the sample.

Total lengths - is the result of the quotient of the determined distance and the length of the swimming pool (25 m). The result is divided by the number of LEDs, and the calculated value is converted to milliseconds.

LED charge time - is the result of technical parameters of the LEDs used, which amounts to $15 \mathrm{~ms}$ for the entire tape of 250 LEDs. For example, if you determine $100 \mathrm{~m}$ to overcome during the 80 seconds, the system will calculate the cycle time of the refresh location LED light at $65 \mathrm{~ms}$.

\section{Results}

\section{Validation tests of devices}

Over the course of the project three acceptance test sessions were scheduled. The first of these, on land, was to verify the integrity of the entire solution, the accuracy of time, and the ergonomic user interface. Two further 
sessions were held in the water with the participation of swimmers. Each session lasted 60 minutes. At this time, the LED tape was at the bottom of the swimming pool. More test cases are described below.

\section{Assessment of the level of brightness of the LED light and its visibility in the aquatic environment}

Each of the swimmers is paired to a specific color displayed on the LED tape. At the stage of the assumptions of the project, it was decided that a given swimmer would be represented by three physical LEDs. During the tests, based on interviews with swimmers it was learned that the visibility and perception of the light in the aquatic environment was very good.

\section{Evaluation of the LED tape seal in an aquatic environment}

The LED tape was sealed and placed on the bottom of the swimming pool (depth of 1.4 to $1.8 \mathrm{~m}$ ) for the duration of the test session (2 times 60 minutes). After removing the tape from the water a thorough analysis was conducted for leaks. There were no cases of water penetration to the interior of the LED tape.

\section{Assessment of the level and form of the power system}

Power consumption for one RGB LEDs is approx. $60 \mathrm{~mA}$. The design of the project was based on a maximum number of 5 swimmers. Each swimmer is represented by 3 RGB LEDs. The following are estimated on current consumption of the LED tape:

$$
60 \mathrm{~mA} \times 3 \times 5=900 \mathrm{~mA} \approx 1 \mathrm{~A} .
$$

From the equation it follows that the power source system had to use a current of $1 \mathrm{~A}$. In fact, a $2 \mathrm{~A}$ AC adapter was used. In addition, the system is adapted to be compatible with a battery power gel with a capacity of 7 Ah. This ensures that the device is comfortably able to handle longer use periods (up to 7 hours). It also ensures energy reserves for the future expansion of the system.

Another source of power tested was the mains supply. A mains supply will maintain a constant voltage (12 V) over the entire length of the LED tape so that all LEDs light up with equal intensity.

Tests confirmed that both the AC adapter and battery provide enough electricity to power the entire system. In addition, thanks to the additional mains supply, the voltage drop in the final meters of LED tape was so small that it did not affect the intensity of the light.

\section{Time accuracy}

The tests consisted of comparing the time at which the moving light signal emitted by the SPCS relative to the time which was assessed by the electronic startup system (chronometer) using the Colorado Time System (Colorado Time, USA), with an accuracy of $\pm 200 \mathrm{~ms}$. For the test, the light signal SPCS system and the Colorado Time System were turned on at the same time by a single experimenter. The light signal moved at a predetermined time over a fixed distance. Once the distance was covered the light signal stopped, and at the same time the experimenter stopped the chronometer in the Colorado Time System. Table 1 presents the results of the measurements obtained. 
Table 1. The results of validation test

\begin{tabular}{ccc}
\hline Test 1 & Test 2 & Test 3 \\
\hline Fixed time: $150 \mathrm{~s}$ & Fixed time: $75 \mathrm{~s}$ & Fixed time: $60 \mathrm{~s}$ \\
Fixed distance: $150 \mathrm{~m}$ & Fixed distance: $100 \mathrm{~m}$ & Fixed distance: $50 \mathrm{~m}$ \\
\hline Completion time $(\mathrm{s})$ & Completion time $(\mathrm{s})$ & Completion time $(\mathrm{s})$ \\
\hline 150.4 & 75.1 & 60.6 \\
150.2 & 75.6 & 59.5 \\
149.8 & 75.3 & 59.8 \\
150.3 & 74.6 & 60.5 \\
149.7 & 75.2 & 60.3 \\
149.6 & 75.6 & 60.7 \\
150.3 & 74.6 & 59.6 \\
150.4 & 74.8 & 59.8 \\
$\overline{\mathbf{X}} 150.08(150 \mathrm{~s} 133 \mathrm{~ms})$ & $\overline{\mathrm{X}} 75.10(75 \mathrm{~s} 166 \mathrm{~ms})$ & $\overline{\mathrm{X}} 60.10(60 \mathrm{~s} 166 \mathrm{~ms})$ \\
\hline
\end{tabular}

Based on these results it can be stated that the average time obtained to move the light beam emitted from the system SPCS at a fixed distance $(150 \mathrm{~m}, 100 \mathrm{~m}, 50 \mathrm{~m})$ was to be found within the assumed error of measurement. The measurement error was set at $500 \mathrm{~ms}$. Measurement error for distances of $150 \mathrm{~m}, 100 \mathrm{~m}, 50 \mathrm{~m}$, amounted to $133 \mathrm{~ms}, 166 \mathrm{~ms}$, and $166 \mathrm{~ms}$, respectively. This was determined to be the result of human influence, as the starting and stopping of the chronometer Colorado Time System was performed by a person.

\section{Discussion}

The aim of this study was to validate the new SPCS for the control of swim speed in real time with the aid of visual information. The hypothesis that the SPCS device objectively indicates swimming speed and is suitable for use in training and during empirical research has been confirmed.

This study holds that the arguments for controlling the speed of swimming in real time, i.e. quicker adaptation to physical exercise, a reduction in the physiological cost incurred during the performance of exercises when training, improved swimming technique at high intensity levels, and optimization of the kinematic parameters of the swimming cycle seem to be convincing. As has been shown, an important role of the coach during exercise is to provide the swimmer with information about swim time and intensity of the training (Micklewright et al., 2012; Chinnasamy, St Clair Gibson, Micklewright, 2013).

Usually this information reaches the swimmer by means of verbal messages from the trainer. Control over exercise intensity is typically in the form of verbal information; for example, the time taken to cover the distance is provided at the completion of the test exercise. Often this is too late to make any adjustment to the speed and thus the intensity of the swim. Additionally, environmental conditions create many barriers that interfere in the process of information exchange. In the aquatic environment these include factors such as noise, head being submerged in water, and wearing a swimming cap.

Methods have been developed to improve the quality of information flow in the aquatic environment. An example is wireless communication using Bluetooth or Wi-Fi, which has been used to assess intensity by 
measuring heart rate. Zatoń, Szczepan (2014) have used radio waves for wireless verbal communication with the swimmer in order to improve swimming technique. Turner, Smith, Coleman (2008) reported the swimming rate with the use of an audio system. Gonzalez et al. (2002) and Perez et al. (2009) used a submerged chronometer at the bottom of the pool to transmit swimming time information. Zatoń, Kędrak, Rejman (2016) applied the use of sliding mirrors, mounted on trolleys, along the edge of the swimming pool to transmit visual information to swimmers in an effort to improve their breaststroke technique.

However, these methods cannot provide information on swim speed rates in real time. Numerous studies have recognized visual information as an effective form of communication used to improve the performance of physical activities and which might be used in challenging environments, namely, the aquatic environment (Seat, Wrisberg, 1990; Carroll, Bandura, 1990; Proteau, Isabelle, 2002). Visual information also allows for the ability to transfer information in real time (online) when physical activity is in progress. Thus, in the present study visual information was used in order to control the speed of swimming.

Improvement of athletic performance requires the search for new training methods. To date, various methods have been used to control swimming speed and exercise intensity (Gonzalez et al., 2002; Turner et al., 2008; Perez et al., 2009). The present study uses a new system SPCS which transmits visual information to the swimmer in real time regarding the speed of swimming by means of a beam of light moving along the bottom of the pool. The conducted validation tests revealed that the functionality of the device is established within an error measurement margin of $500 \mathrm{~ms}$. This means that the system objectively indicates swim speed. As such, the system can be used as a support device in training as well as during empirical research.

\section{Conclusions}

During the validation tests the usefulness of the SPCS for the control of swim speed in real time with the aid of visual information was confirmed. SPCS can be used in swimming training and for research purposes. An advantage of the system is that it does not limit the movements of the swimmers, which can have a positive impact on their achievements in the water. Additionally, the device features wireless connectivity possibilities using Bluetooth technology and the ability for the application to function within a number of operating systems (Windows, Android, iOS).

\section{Acknowledgments}

Development of the "Swimming Pace Control System" (SPCS) was funded by a grant from "Young Scientists Research" 65/22/M/2016 and built by Creosiv Jacek Biernacki, PL.

\section{References}

Åstrand, P.O., Rodahl, K., Dahl, H., Stromme, S.B. (2003). Textbook of Work Physiology. Physiological Bases of Exercise (4 ed.). Champaign, Illinois: Human Kinetics.

Bächlin, M., Tröster, G. (2012). Swimming performance and technique evaluation with wearable acceleration sensors. Pervasive and Mobile Computing, 8 (1), 68-81.

Barbosa, T., Keskinen, K., Fernandes, R., Colaço, P., Lima, A., Vilas-Boas, J.P. (2005). Energy cost and intracyclic variation of the velocity of the centre of mass in butterfly stroke. European Journal of Applied Physiology, 93, 519-523. 
Callaway, A.J., Cobb, J.E., Jones, I. (2009). A comparison of video and accelerometer based approaches applied to performance monitoring in swimming. International Journal of Sports Science \& Coaching, 4 (1), 139-153.

Carroll, W., Bandura, A. (1990). Representational guidance of action production in observational learning: A causal analysis. Journal of Motor Behavior, 22, 85-97.

Chinnasamy, C., St Clair Gibson, A., Micklewright, D. (2013). Effect of spatial and temporal cues on athletic pacing in schoolchildren. Medicine \& Science in Sports \& Exercise, 45 (2), 395-402.

Chow, J.W., Carlton, L.G., Ekkekakis, P., Hay, J.G. (2000). A web-based video digitizing system for the study of projectile motion. Physics Teacher, 38, 37-40.

Costill, D.L., Thomas, R., Robergs, R.A., Pascoe, D., Lambert, C., Barr, S., Fink, W.J. (1991). Adaptations to swimming training: influence of training volume. Medicine and Science in Sports and Exercise, 23, 371-377.

Davey, N.P., Anderson, M.E., James, D.A. (2005). An Accelerometer-Based System for Elite Athlete Swimming Performance Analysis. In: S.F. Al Sarawi (ed.), Smart Structures, Devices, and System II (pp. 409-415). Sydney, Australia: Proceedings of the SPIE, 5649 .

Eskofier, B., Kugler, P., Melzer, D., Kuehner, P. (2012). Embedded Classification of the Perceived Fatigue State of Runners: Towards a Body Sensor Network for Assessing the Fatigue State during Running. In: Guang-Zhong Yang (ed.), Proc. 9th International Conference on Wearable Implantable Body Sensor Network (pp. 113-117). London: IEEE.

Fernandes, R.J., Cardoso, C.S., Soares, S.M., Ascensão, A., Colaço, P.J., Vilas-Boas, J.P. (2003). Time limit and VO 2 slow component at intensities corresponding to $\mathrm{VO}_{2} \mathrm{max}$ in swimmers. International Journal of Sports Medicine, 24 (8), 576-581.

Gonzalez, V., Sanchis, E., Villalobos, M., Brizuela, G., Llana, S., Tella, V. (2002). A new electronic system for the control of swimming speed. In: J.C. Chatard (ed.), Book of abstract: Biomechanics and Medicine in Swimming IX (pp. 67-69). France: Publications de l'Université de Saint-Étienne.

Hay, J.G. (2002). Cycle rate, length and speed of progression in human locomotion. Journal of Applied of Biomechanics, 18, $257-270$.

Hohmann, A., Fehr, U., Kirsten, R., Krueger, T. (2008). Biomechanical analysis of the backstroke of the start technique in swimming E-Journal Bewegung und Training, 2, 28-33.

Lees, A. (2002). Technique analysis in sports: a critical review. Journal of Sports Sciences, 20, 813-828.

Micklewright, D., Angus, C., Suddaby, J., St Clair Gibson, A., Sandercock, G., Chinnasamy, C. (2012). Pacing strategy in schoolchildren differs with age and cognitive development. Medicine \& Science in Sports Exercise, 44 (2), 362-369.

Ohgi, Y. (2002). Microcomputer-based acceleration sensor device for sports biomechanics, Sensors, 1, 699-704.

Pelayo, P., Moretto, P., Robin, H., Sidney, M., Gerbeaux, M., Latour, M.G., Marc-Lavoie, J. (1995). Adaptation of maximal aerobic and anaerobic tests for disabled swimmers. European Journal of Applied Physiology Occupational Physiology, 71, 512-517.

Pérez, P., Llana, S., Brizuela, G., Encarnación, A. (2009). Effects of three feedback conditions on aerobic swim speeds. Journal of Sports Science and Medicine, 8, 30-36.

Proteau, L., Isabelle, G. (2002). On the role of visual afferent information for the control of aiming movements toward targets of different sizes. Journal of Motor Behavior, 34 (4), 367-384.

Scruton, A., Baker, J., Roberts, J., Basevitch, I., Merzbach, V., Gordon, D. (2015). Pacing accuracy during an incremental step test in adolescent swimmers. Journal of Sports Medicine, 6, 249-257.

Seat, J.E., Wrisberg, C.A. (1996). The visual instruction system. Research Quarterly for Exercise and Sport, 67, 106-108.

Slawson, S.E., Justham, L.M., West, A.A., Conway, P.P., Caine, M.P., Harrison, R., (2008). Accelerometer profile recognition of swimming strokes. The Engineering of Sport, 7, 81-87.

Szczepan, S., Zatoń, K., Klarowicz, A. (2016). The effect of concurrent visual feedback on the controlled swimming speed. Polish Journal of Sport and Tourism. Polish Journal of Sport Tourism, 23, 3-6.

Tanabe, S., Ito, A. (2007). A three-dimensional analysis of the contributions of upper limb joint movements to horizontal racket head velocity at ball impact during tennis serving, Sports Biomechanics, 6 (3), 418-433.

Toussaint, H.M., Truijens, M. (2005). Biomechanical aspects of peak performance in human swimming. Animal Biology, 55 (1), 17-40.

Turner, A., Smith, T., Coleman, S.G. (2008). Use of an audio-paced incremental swimming test in young national-level swimmers. International Journal of Sports Physiology and Performance, 3, 68-70.

Wilmore, J.H., Costill, D., Kenney, W.L. (2008). Physiology of Sport and Exercise. Champaign, Illinois: Human Kinetics.

Zamparo, P., Pendergast, D., Mollendorf, J., Termin, A., Minetti, A. (2005). An energy balance of front crawl. European Journal of Applied Physiology, 94, 134-144. 
Zatoń, K., Kędrak, M., Rejman, M. (2016). Synchronized feedback (mirror image) and learning of symmetrical movement activities in breaststroke-kick swimming - pilot study. In: S. Szczepan (ed.), Book of Abstract: The 8th International Symposium Science \& Swimming (pp. 54-55). Wroclaw: Ata-Druk.

Zatoń, K., Szczepan, S. (2014). The Impact of Immediate Verbal Feedback on the Improvement of Swimming Technique. Journal of Human Kinetics, 41, 129-137.

Cite this anticle aS: Szczepan, S., Zatoń, K. (2017). Validation of the New Visual Swimming Pace Control System in Real-Time. Central European Journal of Sport Sciences and Medicine, 19 (3), 93-104. DOI: 10.18276/cej.2017.3-09. 